\title{
Improving Layered Graph Layouts with Edge Bundling
}

\author{
Sergey Pupyrev ${ }^{1}$, Lev Nachmanson $^{2}$, and Michael Kaufmann ${ }^{3}$ \\ 1 Ural State University \\ spupyrev@gmail.com \\ 2 Microsoft Research \\ levnach@microsoft.com \\ 3 Universität Tübingen \\ mk@informatik.uni-tuebingen.de
}

\begin{abstract}
We show how to improve the Sugiyama scheme by edge bundling. Our method modifies the layout produced by the Sugiyama scheme by bundling some of the edges together. The bundles are created by a new algorithm based on minimizing the total ink needed to draw the graph edges. We give several implementations that vary in quality of the resulting layout and execution time. To diminish the number of edge crossings inside of the bundles we apply a metroline crossing minimization technique. The method preserves the Sugiyama style of the layout and creates a more readable view of the graph.
\end{abstract}

\section{Introduction}

Layered drawings present directed graphs in a way that the nodes are arranged in horizontal layers. Most approaches for drawing layered graphs follow in practice the algorithm proposed by Sugiyama et al. [14]. This method is intended to produce layouts with a small number of edge crossings and smooth edges. However, when the given graph is dense, even an optimal layout can have numerous edge crossings and edges that are too curved. In fact, the edge clutter quickly makes the drawing useless for understanding the relations among the nodes.

One of the popular methods to improve quality of such drawings is edge bundling [13]9773]. This method can significantly reduce clutter and can also help to highlight high-level edge patterns in a graph. A known shortcoming of the method is that it tends to produce drawings with ambiguity; it becomes hard to visually follow a single edge. Still a good edge bundling improves the readability of a drawing. To the best of our knowledge, our method is the first attempt to apply edge bundling for graphs with multiple layers. The algorithm is relatively simple and fast enough to be used in practice. For dense graphs, in our opinion, the method produces drawings that are easier to analyze than the standard layouts.

The input to our method is the output of the standard Sugiyama algorithm. We deviate some of the edge routes and create edge bundles, thus reducing the clutter. We do not change the positions of the original nodes of the graph, and we preserve the edge homotopy classes relative to the original nodes, i.e. the new routing can be obtained from the previous one by a continuous deformation of the edges without overlapping with the nodes. This visual stability can help the user to analyze the graph, for example, by comparing the original and the bundled drawings. Fig. 11illustrates these ideas and motivates our approach. 


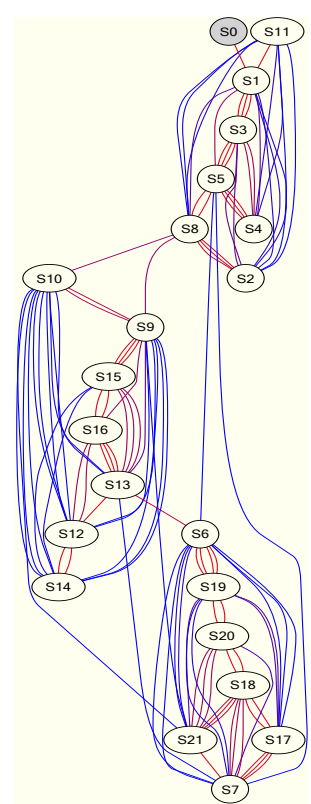

(a) Standard layout

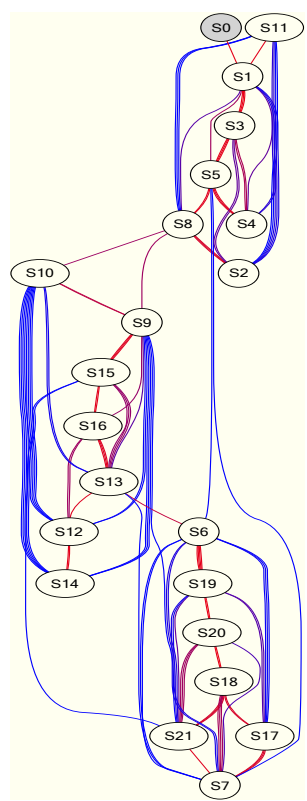

(b) Edge bundling

Fig. 1. The state diagram for the notepad.exe model

Recall that before the Ordering stage of the Sugiyama scheme the original graph is transformed to a proper layered graph. In this graph each original edge is replaced by a connected sequence of proper edges passing through added virtual nodes where a proper edge is an edge between adjacent layers. The first edge of the sequence starts from the original edge source and the last edge ends at the original target.

The starting point of our algorithm is a layered layout of the graph. We change the positions and the order of virtual nodes to organize edges into bundles. On a high level our method consists of the following three stages: create edge bundles of the proper layered graph where each bundle is a subset of edges spanning the same pair of layers; straighten out the edges to avoid excessive bends, and finally reduce the ambiguity in the routing by sorting the edges and drawing them individually inside the bundles.

In Section 3 we give a detailed explanation of our algorithm. Experimental data and results are presented in Section 4 to demonstrate the effectiveness of the method. Finally, Section 5 concludes the paper and discusses additional aspects and future work. The next section summarizes related work.

\section{Related Work}

Sugiyama-style layered layouts were proposed in the early 80's [14]4] and improved in many ways during recent years [8]5]. This method is widely used to layout a graph in a monotone fashion where all edges in a directed-acyclic graph follow the same downward direction. The idea of improving the quality of layered layouts with edge 
bundling is related to edge concentration [13], where a two-layered graph is covered by bicliques reducing the number of drawn edges. Later Eppstein et al. [6] proposed confluent graph drawings, allowing groups of edges to be merged and drawn together.

Recently Holten and van Wijk [9] suggested edge bundling based on an additional tree structure. Unfortunately, not every graph comes with a suitable underlying tree and an artificial one might affect the final layout in an undesirable way. Methods of edge bundling for general graphs were presented by Cui et al. in [3] and by Holten et al. in [10]. We notice that for dense graphs both methods produce ambiguous layouts where it is hard to follow a single edge. In addition, these techniques do not guarantee that the edges are drawn downward which is a requirement of the Sugiyama scheme.

The paper on the metro-line crossing minimization problem by Argyriou et al. [1] inspired us to use the technique of metro-lines crossing minimization to minimize edge crossings inside of the bundles. The paper on circular layouts by Gansner and Koren [7], where bundles were built by minimizing the total ink, also influenced our work.

Unlike the approaches of Holten [10] and Cui [3], we preserve the edge homotopy classes while creating bundles. Moreover, our method takes into consideration the dimensions of the nodes and routes the bundles without overlapping them with the nodes.

\section{Edge Bundling}

We start with some basic definitions. A directed graph $G$ is a pair $(V, E)$, where $V=$ $\{1, \ldots, n\}$ is the set of nodes and $E \subset V^{2}$ is the set of edges. As usual for the Sugiyama algorithm, we may assume that $G$ is an acyclic graph. A layering $L$ of $G$ is a partition of $V$ into sets of layers $L_{1}, \ldots, L_{h}$ such that for every edge $(u, v) \in E$ with $u \in L_{i}$ and $v \in L_{j}$ holds $i<j$. From $G$ and $L$, we build a proper layered graph $G^{p}$ : For each $e=(u, v)$ of $G$ with $u \in L_{i}, v \in L_{j}$ we add to $G^{p}$ nodes $u=d_{i}, d_{i+1}, \ldots, d_{j}=v$, add $d_{k}$ to $L_{k}$ for $k \in[i, j]$ and add to $G^{p}$ edges $\left(d_{k}, d_{k+1}\right)$ for $i \leq k<j$. Nodes $d_{k}$ with $i<k<j$ are called virtual nodes. They are unique for every edge. We denote by $D(e)$ the sequence $d_{i}, d_{i+1}, \ldots, d_{j}$. Nodes of $G^{p}$ which are also nodes of $G$ are called original nodes. Edges of $G$ are called original edges.

The Sugiyama algorithm assigns a point in the plane to each node of $G^{p}$ such that the nodes of the same layer have the same $y$-coordinate. For the purpose of our discussion we assume that the edges are drawn as polylines; for an edge $e$ of $G$ the polyline is defined by the positions of nodes of $D(e)$.

Suppose $S$ is a subset of edges of $G^{p}$ connecting a pair of adjacent layers. We define bundling of $S$ as a procedure where we horizontally shift the ends of the edges of $S$ to make them coincide (Fig. 2(a). After bundling the edges of $S$ together we call $S$ a bundle, the edges of $S$ are called bundled edges, and those nodes that as a result of the shift acquired the same positions are called bundled nodes. We define the graph ink [15] as the sum of the lengths of line segments needed to draw the graph edges.

The order of the nodes on a layer allows us to define an equivalence relation on the layer nodes where each original node is equivalent only to itself, and any two virtual nodes are equivalent if and only if there is no original node between them. Consider two adjacent layers. We subdivide the edges of $G^{p}$ between these two layers into mutually disjoint groups; two edges belong to the same group $G r$ if and only if their sources are equivalent in the upper layer and their targets are equivalent in the lower layer, see 


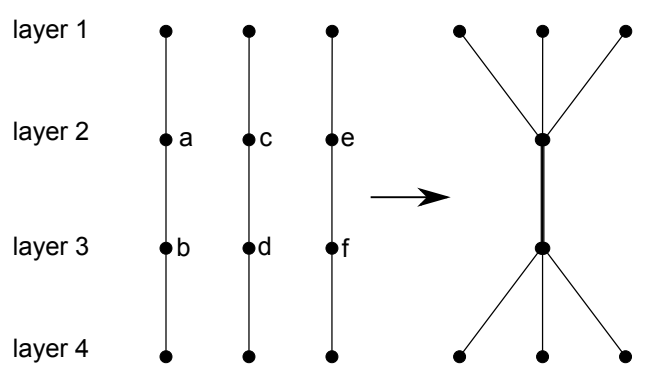

(a)

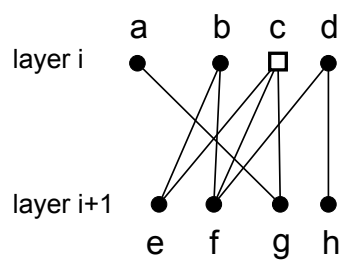

(b)

Fig. 2. (a) Bundling of edges (a,b), (c,d), and (e,f). (b) The original node $c$ divides the edges into three groups: $\{(a, g),(b, e),(b, f)\},\{(c, e),(c, f),(c, g)\}$, and $\{(d, f),(d, h)\}$.

Fig. 2(b) We bundle together some of the edges of the same group if routing them on top of each other saves ink. In order to preserve the homotopy classes of the edges we never bundle together edges from different groups. Note that we will not try to solve the ink minimization problem optimally but propose several greedy heuristics. We address the complexity issue in Section 5 .

\subsection{Identifying Edge Bundles}

We use the output of the Sugiyama layout algorithm as the starting point. More precisely, we are given a layering $L$ and the node positions of graph $G^{p}$.

We process the graph layers downward, consider each group $G r$ separately and bundle together some of the edges in $G r$. Initially, for each edge, we create a bundle containing only this edge. Iteratively we try to find a pair of bundles whose merge maximally reduces the ink, and, if a pair is found, we create a new bundle from the pair components. The group processing stops when the ink does not decrease anymore or there is only one bundle in the group. The procedure on a high level is sketched below.

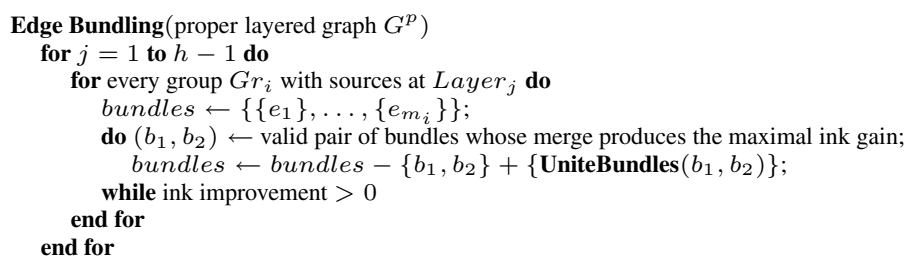

UniteBundles $\left(b_{1}, b_{2}\right)$ returns the union of $b_{1}$ and $b_{2}$. It also shifts the ends of the edges of the union to make the edges coincide. The new coordinates of the edge sources (targets) are set to the average of the coordinates of edge sources (targets) of $b_{1} \cup b_{2}$.

Our experiments show that this strategy alone does not always produce good drawings. In a layout with little ink original edges may be highly curved. To address this issue we restrict the bundling with the following angle constraint:

- Bundling of two edges is allowed only if it does not introduce sharp bends on the polylines of the original edges participating in the bundling. In our implementation we do not allow for an original edge polyline to turn more than on $\pi / 4$ after bundling (Fig. 3(a)). 


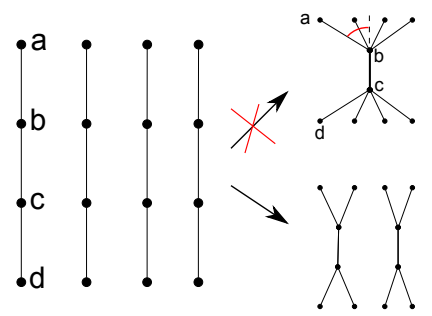

(a)

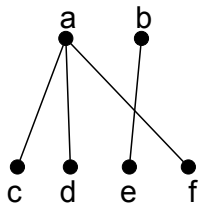

(b)

Fig. 3. (a) A polyline turns more than on $\pi / 4$ at $b$; the bundling is forbidden. (b) Edge $(a, c)$ is compatible only with edge $(a, d)$; edge $(a, d)$ is compatible with edges $(a, c),(a, f)$, and $(b, e)$.

The most expensive part of the bundling scheme described above is the lookup for a suitable pair of bundles whose merge gives the maximal ink gain. We consider three different implementation varying in the time complexity and the quality.

A naive lookup. The brute force approach is to try all the pairs of bundles from the same group $G r_{i}$ of size $m_{i}$ and find a pair whose merge gives the maximal ink gain. Let $t$ be the time needed to calculate the ink gain for the merge of two bundles. In the simplest case when bundles contain only one edge $t=O(1)$. However, in the worst case $t=O(m)$, where $m$ is the number of the edges in $G^{p}$, because moving a bundle involves moving the edges adjacent to the bundle above and below. A performance analysis of processing $G r_{i}$ gives us $O\left(m_{i}^{3} t\right)$ since before each merge we consider all $O\left(m_{i}^{2}\right)$ bundle pairs, we perform $O\left(m_{i}\right)$ merges as the number of bundles is reduced by one at every merge, and each ink gain computation costs $O(t)$ time. That gives us $O\left(m^{3} t\right)$ for all the groups since $\sum m_{i}=m$ and therefore $\sum m_{i}^{3} \leq m^{3}$. Note that this is a worst-case estimation; our experiments show a much better behaviour in practice.

More efficient lookup. The idea here is to avoid calculating ink gain for distant pairs of bundles of the group. For convenience below we denote a bundle by the x-positions of its source and target. For two bundles $e=(x, y)$ and $e^{\prime \prime}=\left(x^{\prime \prime}, y^{\prime \prime}\right)$, we say that $e^{\prime}=\left(x^{\prime}, y^{\prime}\right)$ lies between $e$ and $e^{\prime \prime}$ if and only if both endpoints of $e^{\prime}$ lie between the corresponding endpoints of $e$ and $e^{\prime \prime}$; that is $\min \left(x, x^{\prime \prime}\right) \leq x^{\prime} \leq \max \left(x, x^{\prime \prime}\right)$ and $\min \left(y, y^{\prime \prime}\right) \leq y^{\prime} \leq \max \left(y, y^{\prime \prime}\right)$. This definition includes the case where $e$ and $e^{\prime \prime}$ cross each other (Fig. 3(b) . We call two bundles compatible if there is no other bundle lying between them. We noticed that the Sugiyama scheme usually produces such outputs that if we reduce ink by uniting bundles $e, e^{\prime \prime}$ and $e^{\prime}$ lies in between then we would also save ink bundling $e, e^{\prime}$ and $e, e^{\prime \prime}$. The heuristic here is based on this observation.

We enumerate over all the pairs of bundles from group $G r$ but check the ink gain only for compatible ones. Then we merge a pair of bundles giving the maximal ink gain. In the worst case group $G r_{i}$ contains $O\left(m_{i}^{2}\right)$ compatible pairs. In practice we found that only a small fraction of all possible pairs are compatible. By more involved arguments, it can be seen that the number of compatible pairs is $O\left(m_{i}+c r\right)$, where $c r$ denotes the number of edge crossings within group $G r_{i}$. As a result, we compute the ink gain rarely comparing with the naive implementation, and obtain a significant speedup.

Fast restricted lookup. In the third heuristic we avoid enumerating over all bundle pairs of a group but only consider compatible pairs having a common endpoint. 


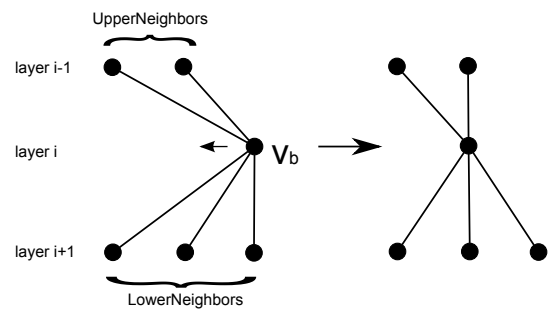

(a)

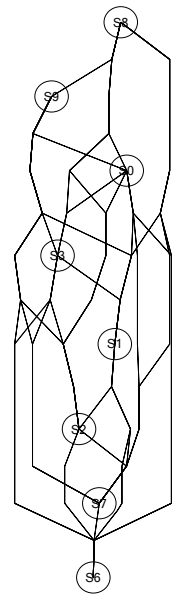

(b)

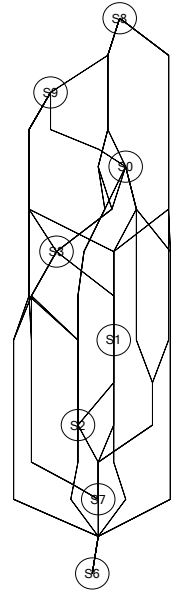

(c)

Fig. 4. (a) Shift of node-set $V_{b}$ results in edges with less turns. (b) Graph layout before straightening phase. (c) Graph layout after straightening phase.

For each node $v$ we order its adjacent edges $\left(v, u_{i}\right), 1 \leq i \leq d_{v}$ by the $x$-coordinates of the targets. Here $d_{v}$ is the out-degree of the node. Then we compute the ink gain of bundling each pair $\left(v, u_{i}\right),\left(v, u_{i+1}\right), 1 \leq i<d_{v}$ and store these values in a balanced tree. A pair giving the maximal ink gain is merged to a bundle. Ink gains of the pairs referencing merged bundles are removed from the tree. Ink gains of merging the new bundle with its left and right neighbors, if they exist, are inserted into the tree. When merging does not decrease the ink, or there is only one bundle left, we stop the procedure and proceed to the next node of the layer. To process the whole graph we sweep top-down layer-by-layer and bundle only pairs with the common source. After the first pass we sweep bottom-top and merge only pairs with a common target.

Processing node $v$ takes $O\left(t d_{v} \log d_{v}\right)$ steps, where $t$ is the time to compute the ink gain. Note that the ink gain calculation might be cheaper for this special case than in general since we move only one end of the bundle. Processing group $G r_{i}$ requires $O\left(t m_{i} \log m_{i}\right)$ steps. Summing over the groups, we achieve a bound of $O(t m \log m)$.

In section 4 we give more details of quality and practical performance of the suggested heuristics.

\subsection{Straightening Edges}

The polylines generated by the method described above often have too many sharp turns. To resolve this problem we use a modification of the median heuristic [8[5], producing smoother and more vertically aligned edges. We iterate over the graph layers in upward direction and try to shift each set of bundled nodes to align its position with the median position of its neighbors from the layer below (Fig. (4).

The shift follows two rules: (I) the set of bundled nodes is shifted only if the new position is outside of the shapes of the original nodes and (II) the number of turns of the edge polylines passing through the shifted nodes does not increase. After sweeping the 


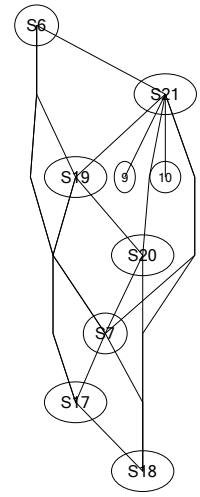

(a)

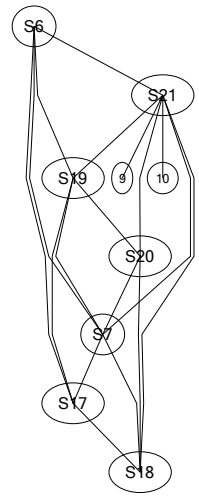

(b)

Fig. 5. Bundled graph before (a) widening with high level of ambiguity and after (b) with optimal crossing number

graph upward we sweep the layers downward. Our approach is sketched below under the assumption that the top layer has index 1.

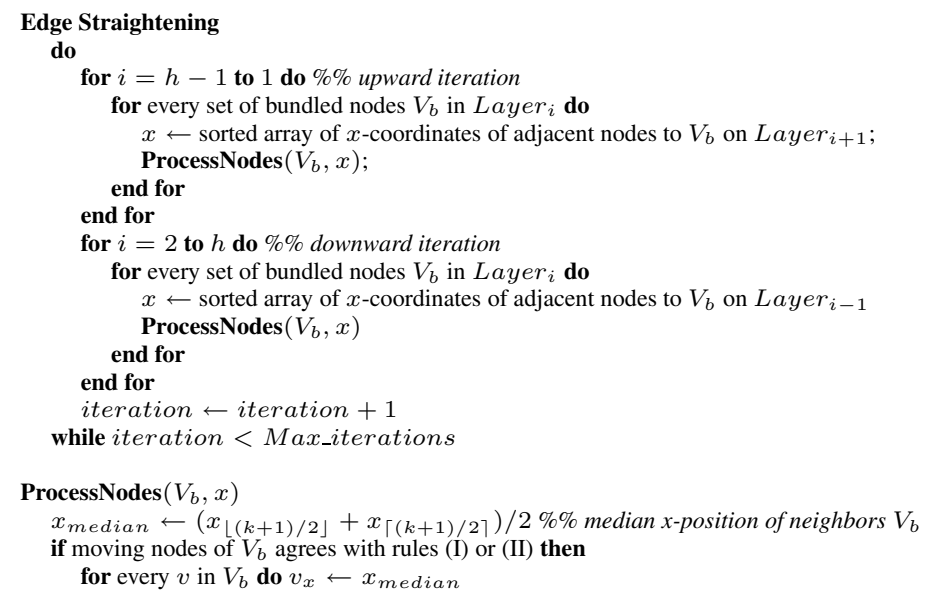

The straightening step requires Max_iterations iterations, which is 10 in our implementation, over the graph nodes. Processing each node takes $O(d \log d)$ steps, where $d$ is the out-degree of the node. Summing over the nodes gives $O(m \log m)$ time. Here we take into account that the sum of all out-degrees of the nodes is equal to $m$.

\subsection{Metro-Map Widening}

After uniting edges we have a bundled graph where the ambiguity is often too high (see Fig 5(a). In this section, we propose a bundle "widening" technique which helps to reduce the ambiguity and highlight the importance of a single bundle.

We draw each edge within the same bundle individually. This is performed by horizontally moving apart coinciding edge ends to slightly separate the bundled edges. 


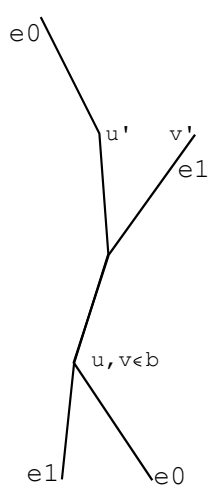

(a)

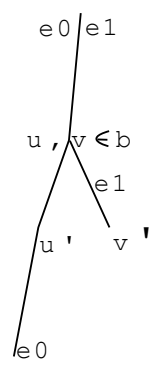

(b)

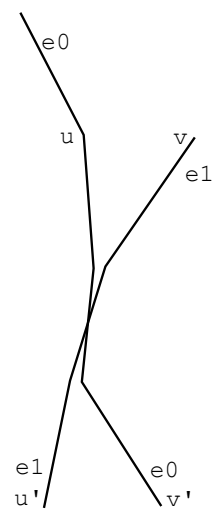

(c)

Fig. 6. (a), (b) $e_{0}<e_{1}$; (c) $e_{0}$ and $e_{1}$ have to cross each other

To avoid introducing unnecessary edge crossings, we order the edges at bundled nodes and spread the nodes according to this order. The problem of finding such orders is similar to the metro-line crossing minimization problem [21].

To define the order, consider two original edges $e_{0}$ and $e_{1}$. Suppose $e_{0}$ has node $u$, and $e_{1}$ has node $v$, which are bundled together to the node set $b$. To define the order between $e_{0}$ and $e_{1}$ at $b$, we first walk up the layers simultaneously over $e_{0}$ and $e_{1}$ starting from $u, v$ until we find nodes $u^{\prime}$ and $v^{\prime}$, which are not bundled together, or reach the source of at least one of the edges. If we find $u^{\prime}$ and $v^{\prime}$, as in Fig 6(a), we order $e_{0}$ and $e_{1}$ at $b$ according to the $x$-coordinates of $u^{\prime}$ and $v^{\prime}$. If such nodes are not found, that is the edges have the same prefix before $b$, we walk down the edges, again looking for the first fork. If not bundled nodes $u^{\prime}$ and $v^{\prime}$ are found, as in Fig 6(b), we order $e_{0}$ and $e_{1}$ at $b$ according to the $x$-coordinates of $u^{\prime}$ and $v^{\prime}$. If no forks are found then two edges belong to the common multi-edge; we order $e_{0}$ and $e_{1}$ at $b$ arbitrarily but keep the same order for every other common bundled node of $e_{0}$ and $e_{1}$.

To use the calculated orders, we uniformly, with some gap given in advance, spread by $x$ the nodes of a bundled node set. We keep the same average of the $x$-positions of the nodes of a set. After widening only unavoidable edge crossings remain. An unavoidable crossing occurs for a pair of original edges $e_{0}, e_{1}$ if there are nodes $u, v, u^{\prime}$, and $v^{\prime}$ such that $u, v$ belong to $e_{0}, u^{\prime}, v^{\prime}$ belong to $e_{1}, u, v$ belong to the same layer, and $u^{\prime}, v^{\prime}$ belong to the same layer, and $\left(x_{u}-x_{v}\right)\left(x_{u^{\prime}}-x_{v^{\prime}}\right)<0$ holds (see Fig. 6(c)). The result of widening is illustrated in Fig. 5(b).

We now discuss the computation cost of this step. The comparison of any two edges can be done in $O(h)$ time, where $h$ is the number of layers. Therefore, the sorting of $k$ edges needs $O(h k \log k)$ time. We need to order the edges at each bundled node set. Let $k_{i}$ be the number of edges passing through the $i$-th bundled node set. Then the total complexity of our algorithm is $O\left(\left(k_{1} \log k_{1}+\ldots+k_{n} \log k_{n}\right) h\right)$, where $k_{1}+\ldots+k_{n}=$ $n$ and $n$ is the number of virtual nodes in the proper layered graph. Here we take into account that through every virtual node passes only one original edge. The maximum of $k_{1} \log k_{1}+\ldots+k_{n} \log k_{n}$ is $n \log n$; it is reached when some $k_{i}=n$. Therefore, the algorithm works in $O(h n \log n)$ steps. 


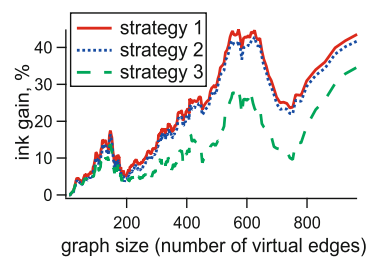

(a) The Rome and North graphs

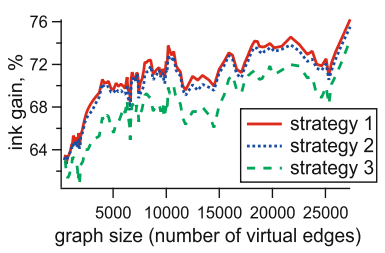

(b) Dense graphs

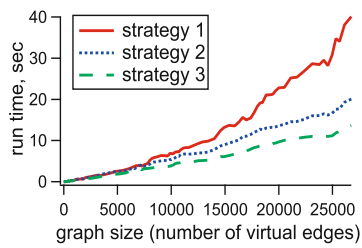

(c) Execution time

Fig. 7. (a-b) The quality of the ink minimization strategies; strategy 1 is the naive lookup, strategy 2 is the efficient lookup, and strategy 3 is the fast restricted lookup. Each data point refers to the average ink improvement of all the graphs in the collection with the same number of edges in the proper layered graph $G^{p}$. (c) shows the comparison of execution times of strategies.

At the final stage we follow[12]; we insert new points into the polylines to avoid edge-node overlaps, and beautify each polyline by fitting cubic Bezier segments in its corners. We do not have an estimate of the complexity of this step, but in practice it takes only an insignificant fraction of the whole time.

\section{Experiments}

We implemented our method in tool MSAGL [12], and used the tool in our experiments. We applied the edge bundling to some real-world graphs and three graph collections:

- Rome [16] contains 11528 graphs with 10 - 100 nodes and 9 - 158 edges. They are obtained from a basic set of 112 real-world graphs. The graphs are originally undirected; we orient the edges according to the node order given in the input files.

- North [16] contains 1175 graphs grouped into 9 sets, where set $i=1, \ldots, 9$ contains graphs with $10 i$ to $10 i+9$ edges.

- Dense is a collection of power-law graphs as used in the social network analysis. The collection contains 116 graphs with $65-95$ nodes and $350-850$ edges; it was generated with the benchmark framework described in [11].

The performance and quality analysis of three edge bundling schemes is presented at Fig. 7. It shows the comparison of the ink minimization strategies; the average run-time and the ink gain for the graphs having the same number of virtual edges. The experiments have been run on an Intel Core-2 Duo $1.83 \mathrm{GHz}$ with 2GB RAM. The simplest implementation reduces the total ink by four times within 40 seconds for the graphs with 25000 virtual edges. The second strategy works two times faster, while its quality of the ink minimization is almost the same. The implementation with restrictions is the fastest: our largest graphs are processed within $10-12$ seconds. Typical layouts obtained with different ink minimization schemes are demonstrated by Fig. 8. We conclude that the second and third strategies are both practical. The former is more aggressive: it bundles larger amount of edges and creates less cluttered images. The latter bundles the edges with common sources or targets only, and thus produces the layouts with less ambiguity.

We compared the time complexity of the edge bundling with other stages of the Sugiyama algorithm. It can be seen that building of bundles is the most time-consuming 


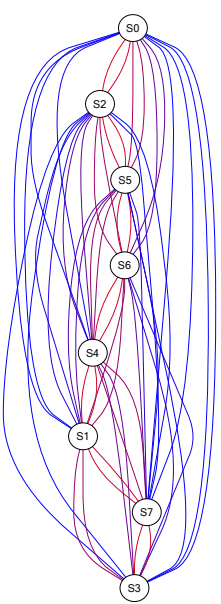

(a)

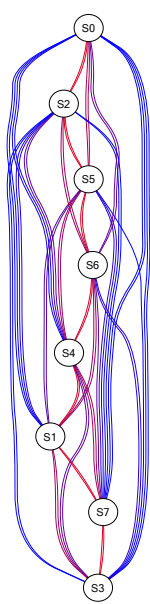

(b)

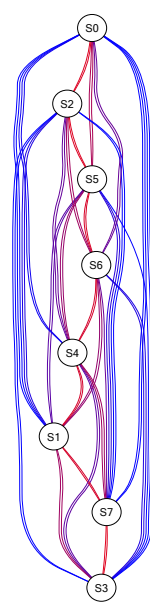

(c)

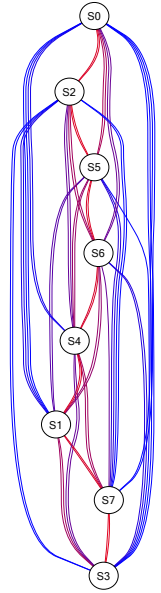

(d)

Fig. 8. A comparison of bundle strategies. (a) The standard layout. (b) The naive lookup. (c) The efficient lookup. (d) The fast restricted lookup.

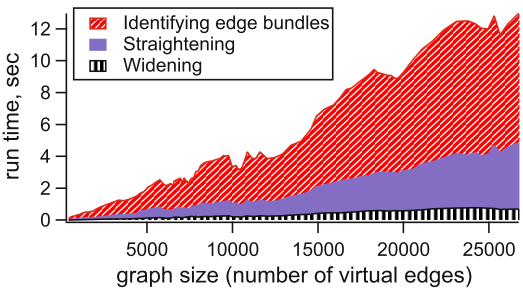

(a)

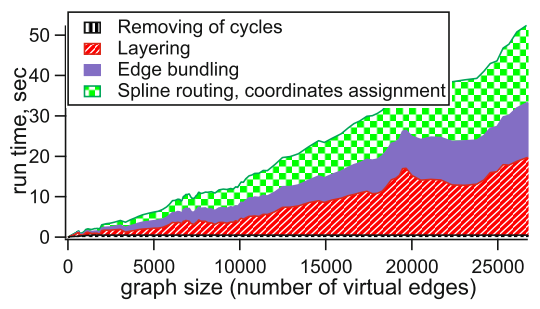

(b)

Fig. 9. Execution time of algorithm stages

part of the edge bundling (Fig. 9(a)p. This step requires around $65 \%$ of the total time. In the whole, the edge bundling time is comparable with the time required by other Sugiyama stages as layering and $x$-coordinate assignment (Fig. 9(b)). The analysis of performance shows that our algorithm handles well medium-sized graphs containing up to several hundred nodes and a thousand edges.

A real-world example of layered layout is state machine diagram. A tool [17] created a model of the standard Windows application notepad.exe. We applied our method to show the state machine of the model. The nodes of the graph depict all possible states of the Notepad model and the edges correspond to the user actions like adding/deleting characters, selecting text, etc. The original and bundled layouts are shown in Fig. 1 Our method reveals the edge patterns and clarifies the structure of the state machine. Another example of edge bundling is given in Fig. 10

We conclude that our technique is beneficial for dense graphs. For such graphs we utilize the white space better, and the bundles help to visualize the high-level edge 


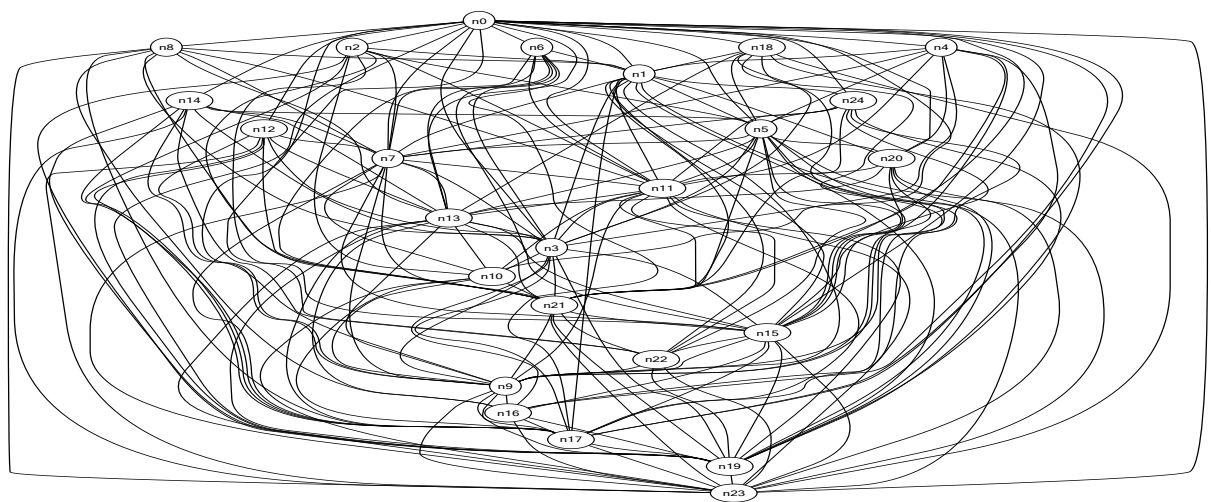

(a)

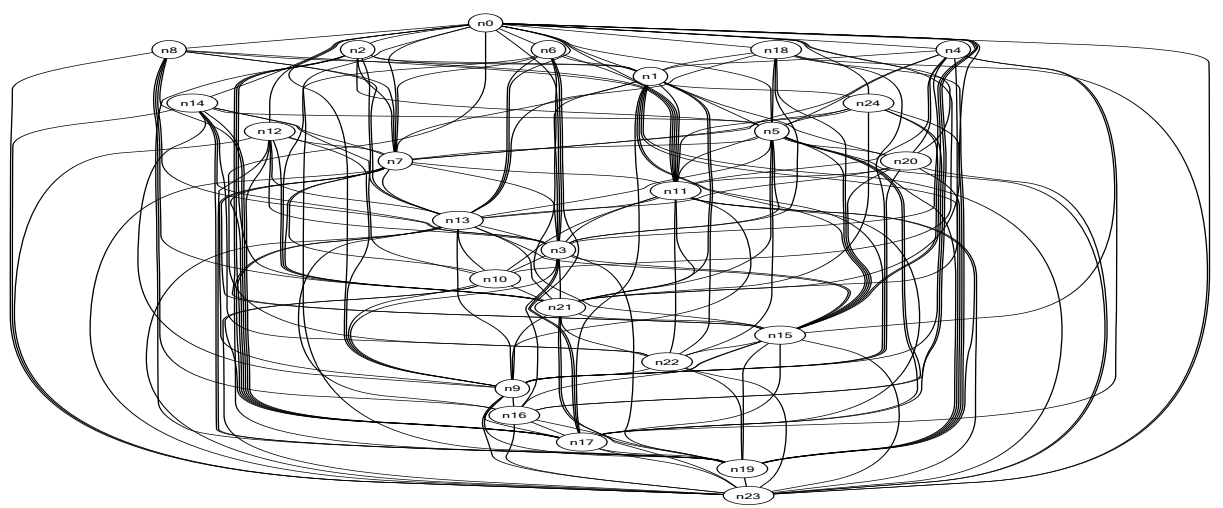

(b)

Fig. 10. Graph from the North collection with 25 nodes and 184 edges

patterns. Our method might introduce some ambiguity as, for example, for edges between $S 19$ and $S 7$ in Fig. 1(b), interactive edge highlighting can help here.

\section{Conclusion, Discussion and Future Work}

We have presented an edge bundling scheme for layered graphs that preserves the initial node positions and the homotopy classes of the edges. It ensures that nodes do not overlap with the edge bundles and that the resulting bundles are relatively straight, making them easy to follow visually. The resulting layout highlights the edge routing patterns and shows significant clutter reduction. Our method can improve layouts produced by other layered graph layout algorithms. The experiments show that the method is fast enough to process medium-sized graphs.

We found yet another application of our method to the Sugiyama algorithm, as a post-processing step for the crossing minimization of the Ordering phase. The idea is for each layer to bundle all virtual nodes which are not separated by an original node. After that we apply the widening technique described in Section 3.3 . This results in an 
order with the minimum number of the edge crossings subject to the given order of the original nodes. In some cases we found up to $30 \%$ of edge crossings reduction.

There are several directions for future work. One could be a global multi-layer bundling processing the edges by taking into consideration the whole graph. We expect to receive drawings of a better quality by analyzing edges globally. Performance of our method depends on the number of virtual edges which can be large and vary significantly for graphs of the same size. The multi-layer approach might lead to an algorithm which does not depend on the number of virtual nodes so heavily. Finally, we would like to understand whether the problem of ink minimization is solvable in a polynomial time. It would be interesting to know how close the ink of drawings produced by our methods is to the minimal ink.

\section{References}

1. Argyriou, E., Bekos, M.A., Kaufmann, M., Symvonis, A.: Two polynomial time algorithms for the metro-line crossing minimization problem. In: Tollis, I.G., Patrignani, M. (eds.) GD 2008. LNCS, vol. 5417, pp. 336-347. Springer, Heidelberg (2009)

2. Bekos, M.A., Kaufmann, M., Potika, K., Symvonis, A.: Line crossing minimization on metro maps. In: Hong, S.-H., Nishizeki, T., Quan, W. (eds.) GD 2007. LNCS, vol. 4875, pp. 231-242. Springer, Heidelberg (2008)

3. Cui, W., Zhou, H., Qu, H., Wong, P.C., Li, X.: Geometry-based edge clustering for graph visualization. IEEE Transactions on Visualization and Computer Graphics 14, 1277-1284 (2008)

4. Eades, P., Sugiyama, K.: How to draw a directed graph. Journal of Information Processing 14(4), 424-437 (1990)

5. Eiglsperger, M., Siebenhaller, M., Kaufmann, M.: An efficient implementation of sugiyamas algorithm for layered graph drawing. Journal of Graph Algorithms and Applications 9(3), 305-325 (2005)

6. Eppstein, D., Goodrich, M.T., Meng, J.Y.: Confluent layered drawings. In: Pach, J. (ed.) GD 2004. LNCS, vol. 3383, pp. 184-194. Springer, Heidelberg (2005)

7. Gansner, E.R., Koren, Y.: Improved circular layouts. In: Kaufmann, M., Wagner, D. (eds.) GD 2006. LNCS, vol. 4372, pp. 386-398. Springer, Heidelberg (2007)

8. Gansner, E.R., Koutsofios, E., North, S.C., Vo, K.-P.: A technique for drawing directed graphs. IEEE Transactions on Software Engineering 19(3), 214-230 (1993)

9. Holten, D.: Hierarchical edge bundles: Visualization of adjacency relations in hierarchical data. IEEE Transactions on Visualization and Computer Graphics 12(5), 741-748 (2006)

10. Holten, D., van Wijk, J.J.: Force-directed edge bundling for graph visualization. In: Computer Graphics Forum, vol. 28, pp. 983-990 (2009)

11. Lancichinetti, A., Fortunato, S., Radicchi, F.: Benchmark graphs for testing community detection algorithms. Physical Review E 80(1) (2008)

12. Nachmanson, L., Robertson, G., Lee, B.: Drawing graphs with GLEE. In: Hong, S.-H., Nishizeki, T., Quan, W. (eds.) GD 2007. LNCS, vol. 4875, pp. 389-394. Springer, Heidelberg (2008)

13. Newbery, F.J.: Edge concentration: A method for clustering directed graphs. In: Proc. 2nd Int. Workshop on Software Configuration Management, pp. 76-85 (1989)

14. Sugiyama, K., Tagawa, S., Toda, M.: Methods for visual understanding of hierarchical system structures. IEEE Trans. on Systems, Man, and Cybernetics 11(2), 109-125 (1981)

15. Tufte, E.R.: The Visual Display of Quantitative Information. Graphics Press, CT (1983)

16. The AT\&T graph collection. http: //www. graphdrawing.org

17. http://research.microsoft.com/en-us/projects/specexplorer 